Article

\title{
On-Chip Cell Incubator for Simultaneous Observation of Culture with and without Periodic Hydrostatic Pressure
}

\author{
Mitsuhiro Horade ${ }^{1, *}$, Chia-Hung Dylan Tsai ${ }^{2}$ (i) and Makoto Kaneko ${ }^{1}$ \\ 1 Department of Mechanical Engineering, Osaka University, Suita 565-0871, Japan; \\ mk@mech.eng.osaka-u.ac.jp \\ 2 Department of Mechanical Engineering, National Chiao Tung University, Hsinchu 30010, Taiwan; \\ dylantsai@nctu.edu.tw \\ * Correspondence: horade@mech.eng.osaka-u.ac.jp; Tel.: +81-6-6879-7333
}

Received: 12 January 2019; Accepted: 15 February 2019; Published: 17 February 2019

\begin{abstract}
This paper proposes a microfluidic device which can perform simultaneous observation on cell growth with and without applying periodic hydrostatic pressure (Yokoyama et al. Sci. Rep. 2017, 7 , 427). The device is called on-chip cell incubator. It is known that culture with periodic hydrostatic pressure benefits the elasticity of a cultured cell sheet based on the results in previous studies, but how the cells respond to such a stimulus during the culture is not yet clear. In this work, we focused on cell behavior under periodic hydrostatic pressure from the moment of cell seeding. The key advantage of the proposed device is that we can compare the results with and without periodic hydrostatic pressure while all other conditions were kept the same. According to the results, we found that cell sizes under periodic hydrostatic pressure increase faster than those under atmospheric pressure, and furthermore, a frequency-dependent fluctuation of cell size was found using Fourier analysis.
\end{abstract}

Keywords: on-chip cell incubator; periodic hydrostatic pressure; periodic pressure; time-lapse observation; cell growth; simultaneous multiple chamber observation

\section{Introduction}

There are many studies addressing cellular responses under mechanical and chemical stimulations [1-7]. Stimulation can be beneficial for cultured cells. For example, Di Carla et al. used caffeine as a xenobiotic stress-inducing agent and found the cell survival rate is promoted under such a chemical stress [8]. Some works used stimulations as a tool for determining cellular properties or physiologies. For example, Seo et al. showed the relationship between mechanical stimulation and the physiologies in a dystrophic heart [9]. Ito et al. applied mechanical stress to red blood cells (RBCs) for different durations and observed a 100 times difference of the time constant in their shape-recovery curves when the stress duration was just increased from $10 \mathrm{~s}$ to $180 \mathrm{~s}$ [7]. Sakuma et al. proposed a cell stress test by moving a RBC back and forth across a microfluidic constriction until the RBC eventually lost its deformability and used it as an index of RBC deformability [10]. There are also studies investigating cell alignment under stimulations. For example, Teramura et al. demonstrated that mechanical stimulation to human iPS cells altered the alignment of actin fibers as well as the expressions of the pluripotent related genes [11]. Subramony et al. investigated the role of nanofiber matrix alignment and mechanical stimulation on mesenchymal stem cell (MSC) differentiation [12]. While there are approaches using 3D printing for aligning cells [13-15], stimulation seems to be a more natural approach, since each cell can choose its comfortable position and orientation with fewer constraints.

Culture with periodic hydrostatic pressure here refers to a periodic stimulation of mechanical stress onto cells during cell culture. The concept of such a periodic hydrostatic pressure is can be 
explained with the analogy to the movement during a human workout. An interesting result was recently presented by Yokoyama group, who utilized periodic pressure during culturing smooth muscle (SMC) cells. They found an optimum recipe regarding culturing elastic cell sheet, and the applied frequency, the minimum pressure, and the maximum pressure of the recipe were $0.002 \mathrm{~Hz}$, $110 \mathrm{kPa}$, and $180 \mathrm{kPa}$, respectively [16]. Under such an optimum recipe, they succeeded in growing a 10-layer cell sheet in 20 days. Furthermore, they made a vascular graft, with a diameter of $1 \mathrm{~mm}$, from the cell sheet and transplanted the graft into a rat for reconnecting a cut artery. The results showed that the rat could continuously survive for 2.5 months after the transplantation, and new capillaries were found grown on the transplanted tissue. This is a sign showing that the body can successfully adapt to transplanted tissue and apply the necessary nutrition to the part.

The first prototype of on-chip cell incubator was recently presented with a result showing that the cells cultured with periodic hydrostatic pressure have a greater number of stress fibers [17]. While cell sheet cultured under periodic hydrostatic pressure is having a greater elasticity than that under atmospheric pressure [16], it is still not clear when and how this difference happens. The main goal of this work is to reveal the difference between cultured cells with and without periodic hydrostatic pressure during the culture. We focused on the cell behavior in the first one hour after cell seeding. The cell behavior was observed with time-lapse images taken from the observation window of a developed on-microscope incubation system. The proposed cell incubator was with two independent culture arrays in it. The key advantage is that we can compare both cell responses under the same conditions except periodic hydrostatic pressure. Through the experiments, we found that cells showed remarkable size growth under periodic hydrostatic pressure with the frequency-dependent fluctuation that the cell size changed with the applied pressure.

\section{Materials and Method}

\subsection{Periodic Hydrostatic Pressure and Optimum Stimulus}

Figure 1a illustrates the analogy of human exercise to cell culture with periodic hydrostatic pressure. The upper and lower figures in Figure 1a are examples of human exercise with weight lifting and cell culture with periodic hydrostatic pressure, respectively. Figure 1b,c explains how cells are cultured under atmospheric pressure and under periodic hydrostatic pressure. The rightmost pictures of Figure $1 b, c$ are our preliminary results of cultured cells. In the results, stress fibers, the actin filaments, were stained with Acti-stain 488 Fluorescent phalloidin for the convenience of observation. It can be seen that the stress fibers of the cells under periodic hydrostatic pressure are thicker than those under atmospheric pressure. The results in Figure 1b,c show clear difference with and without periodic hydrostatic pressure.
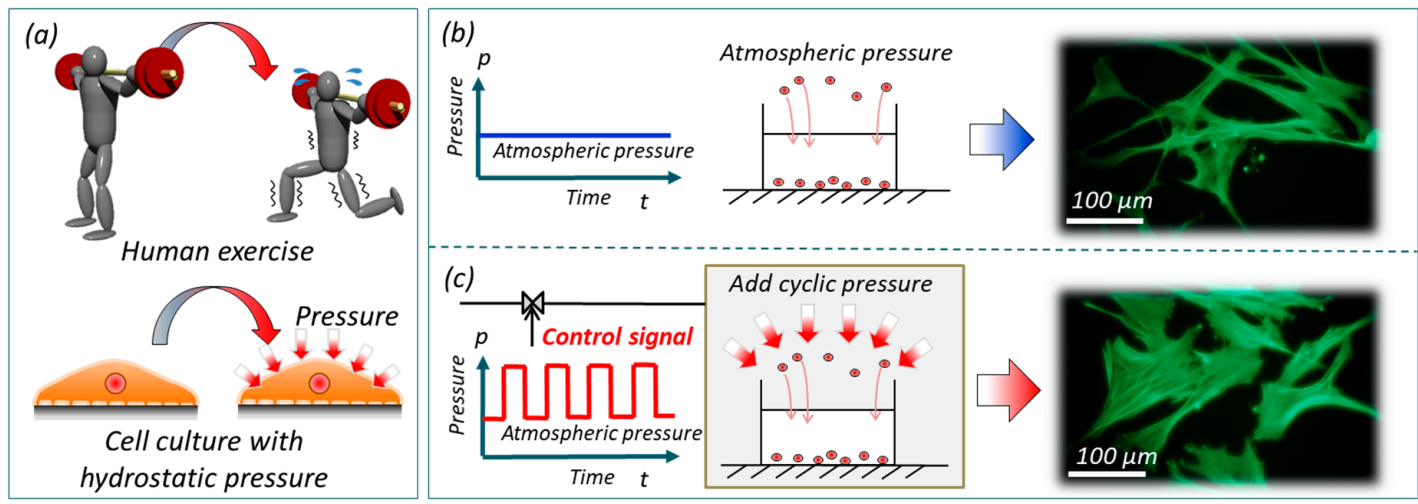

Figure 1. An overview of Periodic hydrostatic pressure. (a) The analogy of Periodic hydrostatic pressure to human exercise. (b) Conventional cell culture and a result showing the stress fibers of the cultured human smooth muscle cells (HSMCs) under atmospheric pressure. (c) Cell culture with Periodic hydrostatic pressure and a result showing the stress fibers of the cultured HSMCs under periodic pressure. The stress fibers, the actin filaments, were stained with Acti-stain 488 Fluorescent phalloidin. 
The three most critical parameters of periodic hydrostatic pressure, are the maximum pressure, the minimum pressure, and frequency. These three parameters control the pressure pattern with respect to time during cell culture. In conventional works of pressure-based stimuli culture, the frequencies of periodic pressure are mostly around $1 \mathrm{~Hz}$, just like heartbeat and breathing [16,18-21]. Different from those studies, we tested low frequency zone much less than $1 \mathrm{~Hz}$ and reached a new optimum frequency, which had not yet been found previously. As for the determination of the pressure pattern, we had to determine both the maximum and minimum pressures in addition to the frequency. It is well known that our blood pressure is slightly higher than atmospheric pressure, it is about $100 \mathrm{kPa}$. When the minimum value of blood pressure, which is also known as diastolic blood pressure, is roughly $80 \mathrm{mmHg}$, about $10 \mathrm{kPa}$. Thus, we set up the lower pressure is $110 \mathrm{kPa}$ which corresponds to human minimum blood pressure. As for the maximum pressure, we selected it based on the gene expression, such as Fiburin and Lysyl [16]. Fiburin and Lysyl are two important components for stress fibers of human smooth muscle cells (HSMCs), where Fiburin is a key component of elastic fiber growth, and Lysyl helps cross-linking. After preliminary experiments, the maximum pressure of $180 \mathrm{kPa}$, roughly equivalent to $600 \mathrm{mmHg}$ and about five times the blood pressure, was chosen [16]. Overall, the parameters of the maximum pressure, minimum pressure, and frequency were $180 \mathrm{kPa}$, $110 \mathrm{kPa}$, and $0.002 \mathrm{~Hz}$, respectively. The time period of one cycle is about $8 \mathrm{~min}$ and $20 \mathrm{~s}$. This means that the periodic pressure between $180 \mathrm{kPa}$ and $110 \mathrm{kPa}$ is given for every $250 \mathrm{~s}$. Of course, we would like to make frequency lower than this, but it could not be done because the $\mathrm{pH}$ balance is broken due to pressurized $\mathrm{CO}_{2}$. If pressurized continues for long time, $\mathrm{CO}_{2}$ continues to dissolve into the culture solution, and $\mathrm{pH}$ becomes below 5.8, while the optimal cell culture $\mathrm{pH}$ is between 5.8 and 6.2.

\subsection{Development of Cell Incubator and Pressure Control}

We aimed to compare cell behaviors with and without periodic hydrostatic pressure during cell culture in this work. To do this, an important issue was how to simultaneously observe the cell groups. We propose the on-chip cell incubator where we can observe what happens in real-time on a single chip, as shown in Figure 2. The chamber arrays on the chip are placed in symmetry to the center line, and they are connected to two independent microfluidic channels. Through this device, we can impart pressure on cells with specified pressure patterns. Microfluidic devices have often been used for experiments under a microscope [22-24]. For example, Eyer et al. used a microchamber array for single cell isolation and analysis of intracellular biomolecules [25]. Reaction experiments and local irritation experiments on single cells can be performed using a microfluidic chip. The on-chip cell incubator in this method was fabricated using a standard photolithography approach with microelectromechanical systems (MEMS) technology and is made of polydimethylsiloxane (PDMS). The fabrications details of the PDMS chip can be found in Appendix A.
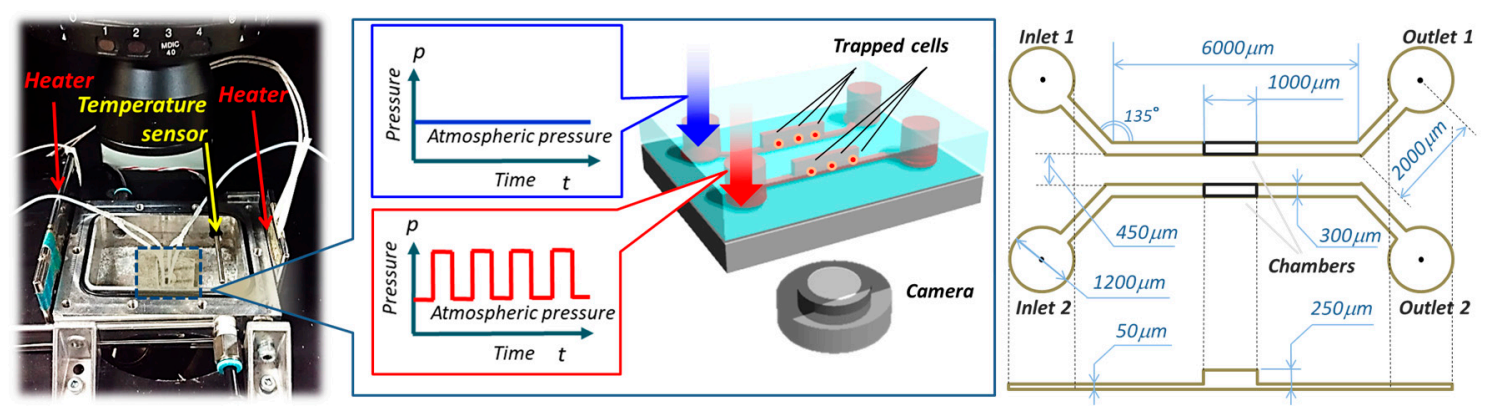

Figure 2. An overview of the experimental system. From left to right are the on-microscope incubation system, design of on-chip cell incubator and its dimensions, respectively. The system is mainly composed of two parallel chambers for simultaneously observation of cell culture with and without Periodic hydrostatic pressure. 
The system is composed of a microscope (OLYMPUS: IX71, Olympus Co., Tokyo, Japan), a commercial incubator (SCA-80DS, ASTEC Inc., Fukuoka, Japan), a compressor (DOP-80SP, ULVAC Inc., Kanagawa, Japan), a flow regulator (ITV2030-212BL, SMC Co., Tokyo, Japan), a pressure sensor (HP101-C31-L50A*B/V1, Yokogawa Electric Co., Tokyo, Japan), a digital CMOS camera (C11440, Hamamatsu Photonics K.K., Shizuoka, Japan), and an on-microscope cell incubation system. The on-microscope incubation system is used for maintaining a culture environment as in the leftmost photo in Figure 2. The temperature around the chip is controlled by a feedback system using a heater and a temperature sensor to maintain an environment of $37^{\circ} \mathrm{C}$. The concentration of $\mathrm{CO}_{2}$ is controlled at $5 \%$ by drawn gas from the commercial incubator to the on-microscope incubation system by the flow regulator. Both the inlet and outlet of the chip are connected with Teflon tubes to the pressure regulator, so that the inside of the micro chambers can be pressurized with a specified level at specified time sequences. As mentioned in the previous section, a pressure of $180 \mathrm{kPa}$ and $110 \mathrm{kPa}$ was applied as the periodic hydrostatic pressure in this work.

\subsection{Chip Preparation and Experimental Procedure}

In order to adhere cells to the PDMS surface for cell culture, $10 \%$ adhesion-assist protein fibronectin was coated on the surface of culture array by injecting $25 \mu \mathrm{L}$ of it from the inlet of the device using a micropipette. The area inside of the PDMS flow channel and micro chamber arrays was filled with the solution and placed at $37^{\circ} \mathrm{C}$ in the incubator for $60 \mathrm{~min}$. Afterwards, the fibronectin solution was removed and rinsed with phosphate buffered salts (PBS).

Human smooth muscle cells (HSMCs) were used for all experiments in this work. Cells were injected into the chamber arrays for seeding from the inlets of the PDMS device. The concentration of HSMC was $2.5 \times 10^{5}$ cells $/ \mathrm{mL}$. Low cell density was used for the convenience of single cell analysis, particularly for avoiding overlaps of the projected area. The tube on the outlet side of the periodic hydrostatic pressure chamber was connected to the compressor for pressurized gas. Since the height of the micro chamber is higher than the other flow micro channel region, more cells can be trapped in the micro chamber due to inertial flow. After cell injection, the cells were gradually attached to the bottom of the glass by gravity force. For the culture with periodic hydrostatic pressure, the tube on the inlet side was sealed and periodic pressure was applied to the chamber. For the culture without periodic hydrostatic pressure, the tube was open to the atmosphere.

Time-lapse imaging was employed for comparing the difference between cell growth with and without periodic hydrostatic pressure. The camera takes a picture covering both chambers simultaneously every $10 \mathrm{~s}$ after seeding. We focused on how the cells grow during the first hour after seeding. Figure 3a shows an example of a captured time-lapse image. Both cells in the two micro chambers were recorded simultaneously, where the upper and lower chamber in Figure 3 are with and without periodic hydrostatic pressure mode, respectively. Only about 5-10 cells were trapped in the chamber of the on-chip cell incubator device for the convenience of single cell observation.

In order to evaluate the degree of cell growth, we introduced the projected area as an evaluation index where it was defined by the projected area of cell in the horizontal plane. An example of cell growth and image processing is demonstrated in Figure 3b,c, respectively. In Figure 3b, cell images were acquired for each record time, and images at $20 \mathrm{~min}, 40 \mathrm{~min}$, and $60 \mathrm{~min}$. Figure $3 \mathrm{c}$ shows the image at $60 \mathrm{~min}$ after seeding and the left shows the original image. The outline in the middle of Figure $3 \mathrm{c}$ allows us to determine the cell area, as shown in the rightmost image in Figure 3c. As a result, we can obtain the information of cell shape and area using image analysis software Image J (1. 50i, Wayne Rasband, National Institutes of Health, USA). 

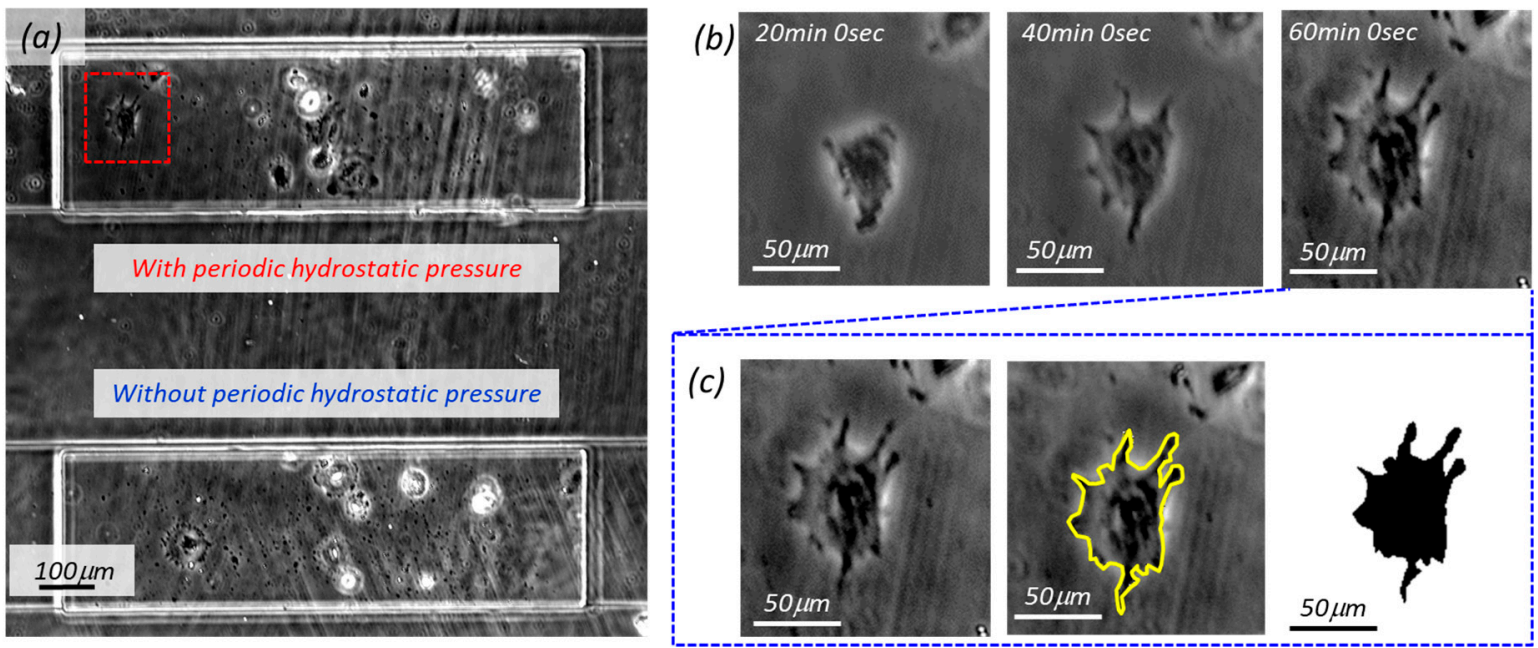

Figure 3. Captured pictures by time-lapse imaging. (a) Captured microscopic image where the chambers on the top and the bottom are the cells cultured with and without the proposed periodic hydrostatic pressure, respectively. An example of cell assessment is demonstrated using the highlighted cell. (b) Selected time-lapse images of the cell at the time of 20,40, and $60 \mathrm{~min}$. (c) The procedure to obtain the extracted cell area.

Figure 4 shows measured results of pressure control during the culture, and the pressure was cycling between $180 \mathrm{kPa}$ and $110 \mathrm{kPa}$ every $250 \mathrm{~s}$. Figure $4 \mathrm{a}, \mathrm{b}$ is close views of increasing and decreasing pressure periods, respectively. From Figure $4 \mathrm{a}, \mathrm{b}$, we can see a reasonably well controlled pressure where an error is roughly less than $1 \%$ with respect to the target value without overshoot. In addition, it can be seen that the time for pressurization to the target values is less than one second.

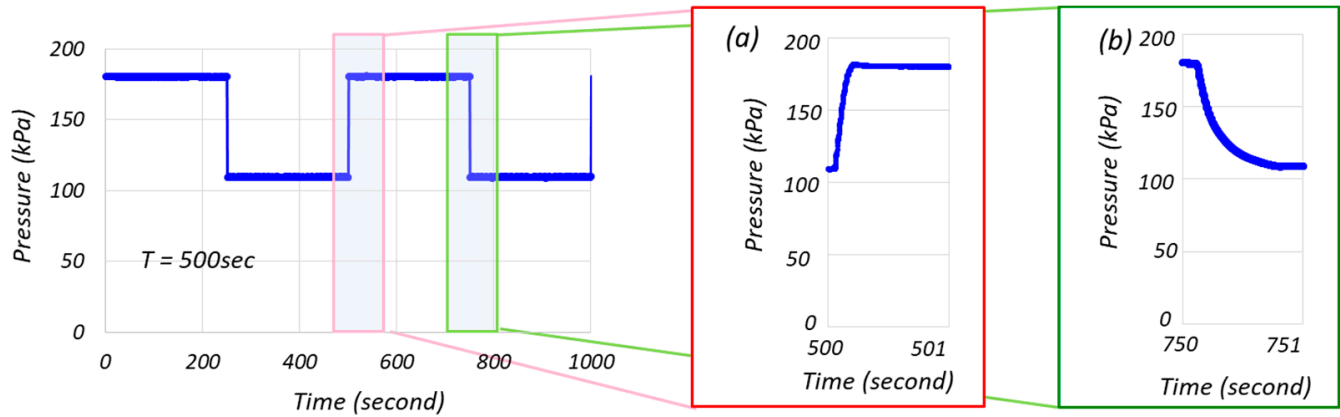

Figure 4. The performance of pressure control during periodic hydrostatic pressure. (a) A close view of the pressure increasing period. (b) A close view of the pressure decreasing period.

\section{Results}

\subsection{Projected Area of Cells with and without Periodic Hydrostatic Pressure}

Figure 5 shows the growth of the projected cell area with respect to time where Figure $5 a, b$ denotes the projected areas and the average value among the six trapped cells, respectively. The cell groups with and without periodic hydrostatic pressure are indicated by red and blue marks and the original of the time axis is the starting time of pressurization. From Figure 5, no significant difference can be seen between the two groups with and without periodic hydrostatic pressure. 

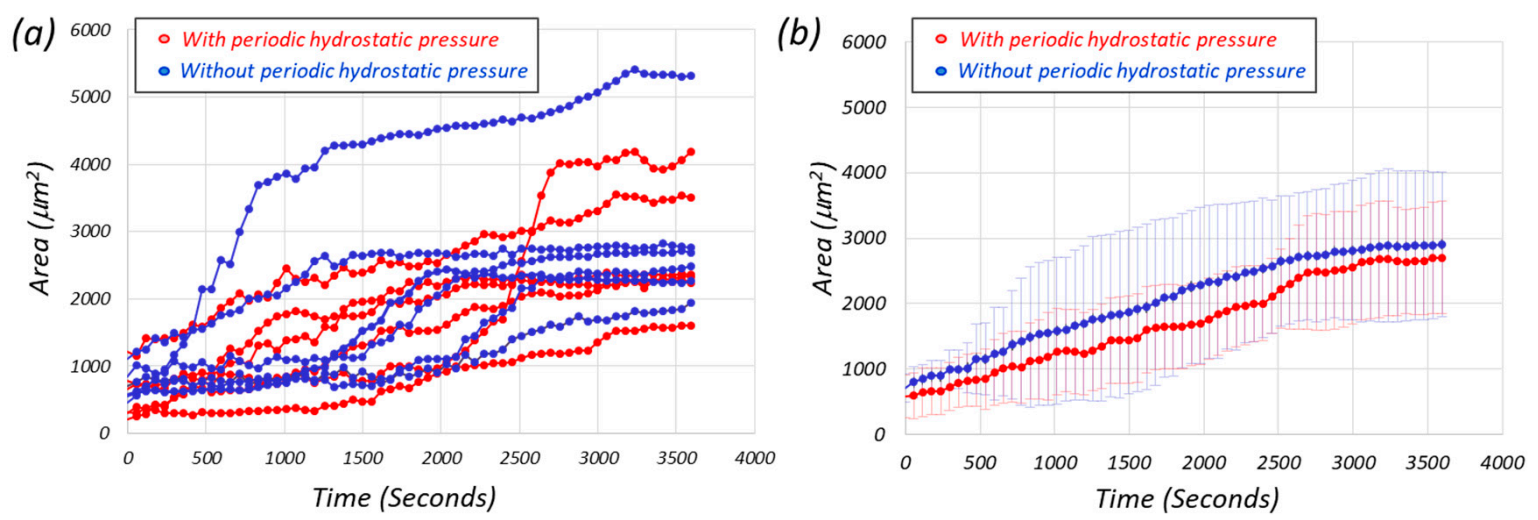

Figure 5. The growth of the projected area with respect to time, where the cell groups with and without periodic hydrostatic pressure are indicated by red and blue marks, respectively. (a) Measured area of six cells from the periodic hydrostatic pressure chamber (red) and 6 cells from control (blue), a culture chamber without periodic hydrostatic pressure (b) The average value and standard deviation from the six cells in each chamber are plotted. No significant difference between two.

An example of cell area changes under periodic hydrostatic pressure is shown in Figure 6, where there is a remarkable point (b). The projected cell area increased rapidly from (b) to (c), and was with nearly three times faster than the initial $15 \mathrm{~min}$. After that, the cell entered another phase where the projected cell area increased with a slightly gentle slope from (c) to (d). It is interesting to know the tendency of the projected cell area with or without periodic hydrostatic pressure with such instances. A time-lapse cell behavior during cell culture can be found in the supplementary material, Videos S1 and S2. Video S1 shows the cell behavior from the point (a) to the point (d), and Video S2 shows the cell behavior after the point (c). After point (c), where rapid growth ended, interesting behavior was observed in which the cell periodically extends in terms of its size.

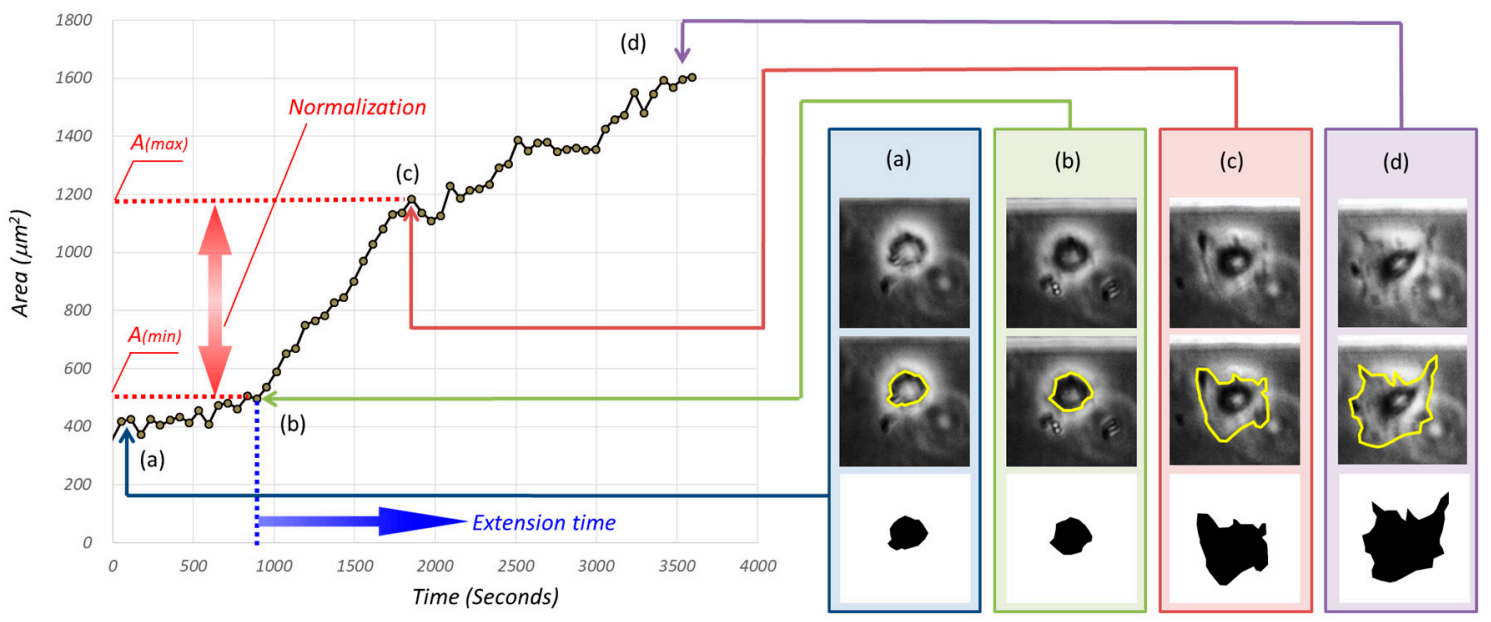

Figure 6. An example of the projected cell area with respect to time. $(\mathbf{a}, \mathbf{b})$ are the cell images at different instance, and from the top to the bottom are the original cell image, contour extraction, the projected cell area, respectively. It should be noted that the increase velocity of the projected cell area from (b) to (c) is larger than that of other phases, such as from (c) to (d). 
For determining point (b) and point (c), we used $S(t)$, defined by:

$$
S(t)=\frac{A_{(t)}-A_{\min }}{A_{\max }-A_{\min }}
$$

where $\mathrm{A}_{\min }, \mathrm{A}_{\max }$, and $\mathrm{A}(t)$ are the projected cell areas at the time in point $(\mathrm{b})$, at the time in point (c), and at the time of $t$, respectively. Simply speaking, point (b) is the starting point when the cell size starts to increase rapidly and point (c) is the ending point when the rapid change of cell size is terminated.

Figure 7 shows the normalized $S(t)$, where the cell groups with and without periodic hydrostatic pressure are indicated by red and blue marks, respectively. The origin of the horizontal axis is the time which corresponds to the point (b) in Figure 6. The velocity of the projected cell area increased dramatically after point (b). From Figure 7 , both cell groups with and without periodic hydrostatic pressure resulted in 1.0 in about $20 \mathrm{~min}(1200 \mathrm{~s})$, which matched well with the definition of $\mathrm{A}_{\min }$ and $A_{\max }$. On the other hand, the tendencies of $S(t)$ shown by the two groups after $20 \mathrm{~min}(1200 \mathrm{~s})$ were different. The cell group with periodic hydrostatic pressure continued to increase the projected cell area with a gentle slope, whereas the cell group without periodic hydrostatic pressure was only a tiny positive slope. The $p$-value of the T test for the cell size of two groups at $20 \mathrm{~min}$ (1200 s) was 0.173 and indicated no significant difference. However, when the time reached $30 \mathrm{~min}(1800 \mathrm{~s})$, the $p$-value became less than 0.05 and demonstrated a significant difference between the growth rate with and without periodic hydrostatic pressure. In other words, the significant difference for the culture with and without periodic hydrostatic pressure happened after the time past $30 \mathrm{~min}$ in Figure 7, as the point (c) in Figure 6.

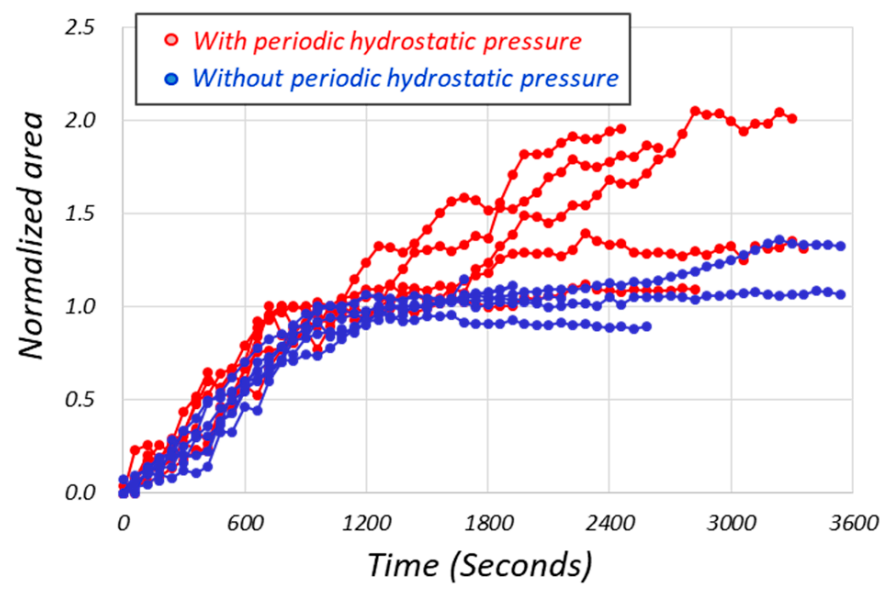

Figure 7. The normalized projected cell area with respect to the time where the origin of the time is redefined by the time corresponding to point (b) in Figure 6. The cell groups with and without periodic hydrostatic pressure are indicated by red and blue marks, respectively, and normalized for each of the six original data shown in Figure 5.

\subsection{Periodic Characteristics in Cell Growth}

Figure 7 shows two important results, one of which is that there is statistically meaningful difference at $30 \mathrm{~min}$ after cell extension, and the other is that the growth pattern is fluctuating with respect to time. To observe the growth pattern more qualitatively, let us rearrange the time domain, so that we can adjust the phase among all experiments under periodic hydrostatic pressure. 
Figure 8 shows the normalized projected cell area $S(t)$, where the origin of the horizontal axis is the time corresponding to (c) in Figure 6, more specifically when the pressure is switched from $180 \mathrm{kPa}$ to $110 \mathrm{kPa}$. This means that $\mathrm{A}_{\max }$ in Equation (1) is the projected cell area at the time $\mathrm{t}$ when the pressure is switched from $180 \mathrm{kPa}$ to $110 \mathrm{kPa}$ in the nearest time around (c) in Figure 6.

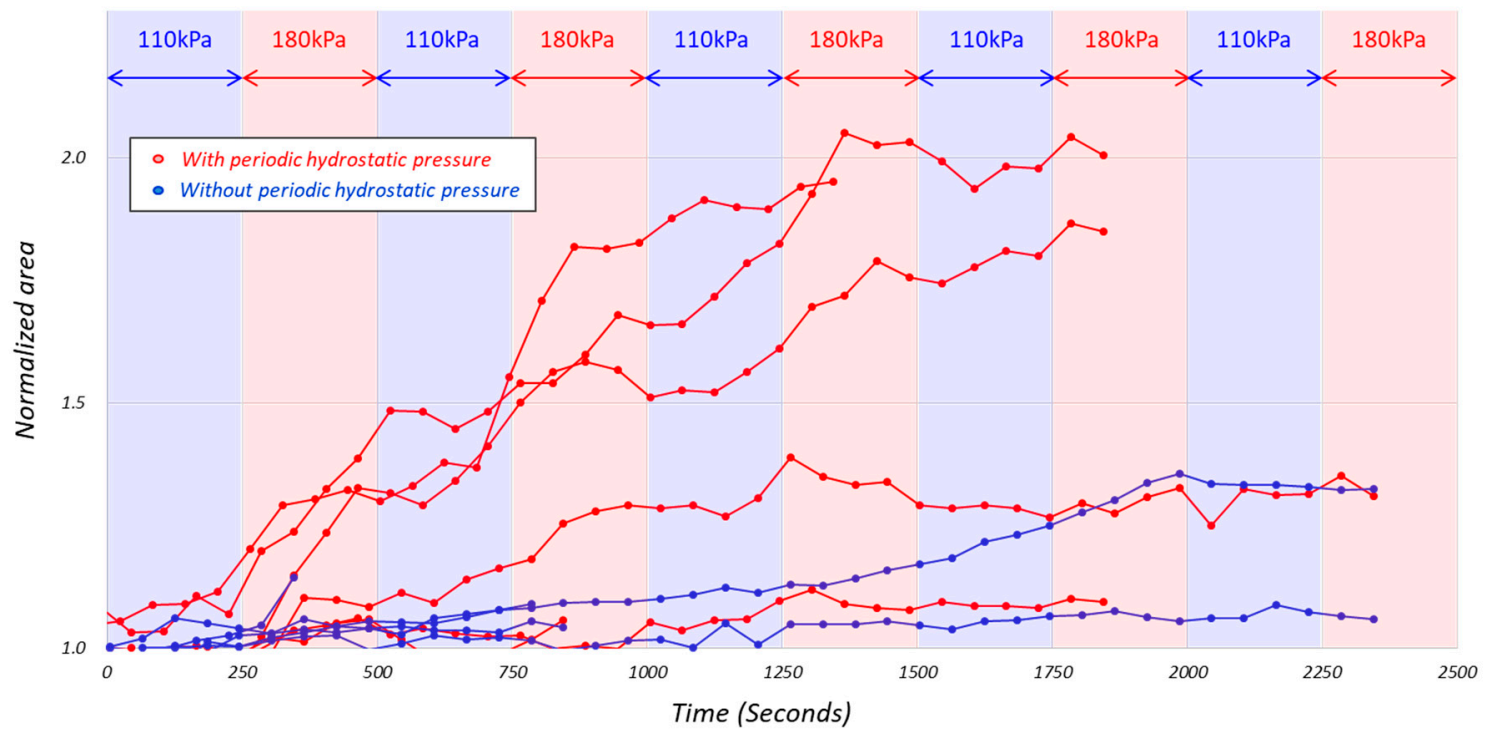

Figure 8. The normalized projected cell area $S(t)$ where the origin of the horizontal axis is the time corresponding to (c) in Figure 6, more specifically when the pressure is switched from $180 \mathrm{kPa}$ to $110 \mathrm{kPa}$ in the nearest time around (c) in Figure 6, and normalized for each of the six original data shown in Figure 5.

\subsection{Frequency Analysis on Projected Area of Cells}

Figure 9 explains how to achieve the frequency analysis for one particular cell under periodic hydrostatic pressure, where Figure $9 \mathrm{a}-\mathrm{c}$ denotes the normalized area with respect to time, the curve defined by $\Delta S(t)=S(t)-S(t)$ approximate curve, and the frequency analysis, respectively. $S(t)$ approximate curve is obtained by a linear fit with the-least-squares method as shown in Figure 9a. From Figure 9c, we can see an interesting observation, namely, frequency-dependent cell growth. The frequency of $0.002 \mathrm{~Hz}$ was a peak of the frequency analysis and it corresponds to the frequency of periodic hydrostatic pressure. Figure $9 c-h$ shows three examples of frequency analysis where (c) through (e) is cultured under periodic hydrostatic pressure and (f) through (h) is cultured under atmospheric pressure. In the cell group cultured under periodic hydrostatic pressure, we can see clear amplitude in the frequency domain with $0.002 \mathrm{~Hz}$, while we cannot see clear amplitude in the frequency domain with $0.002 \mathrm{~Hz}$ in the cell group cultured under atmospheric pressure. 
(a)

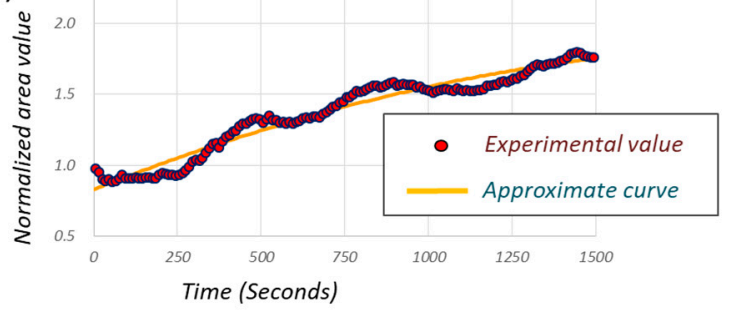

(c)

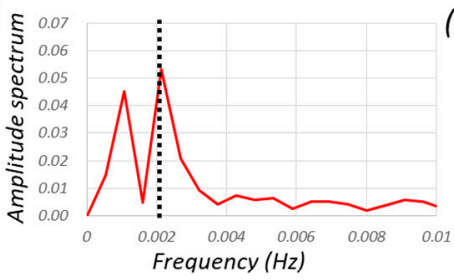

(f)

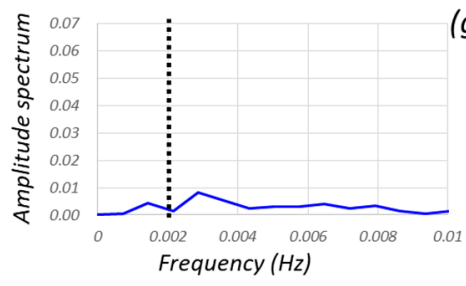

(d)

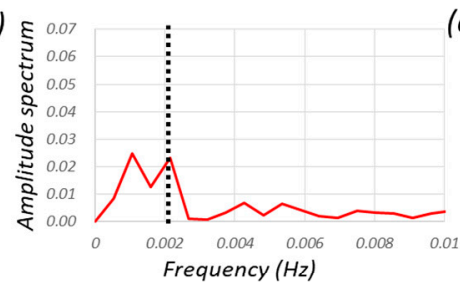

(g)

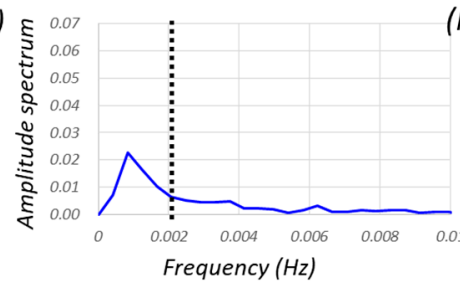

(b)

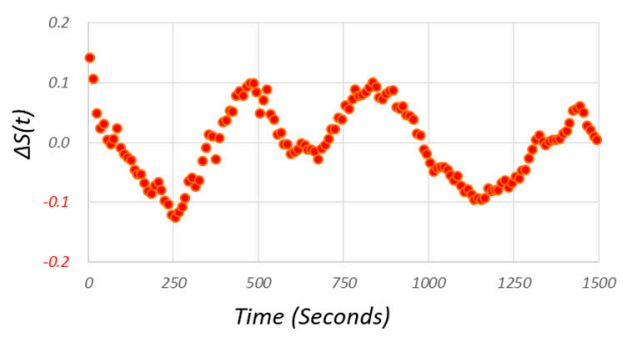

(e)

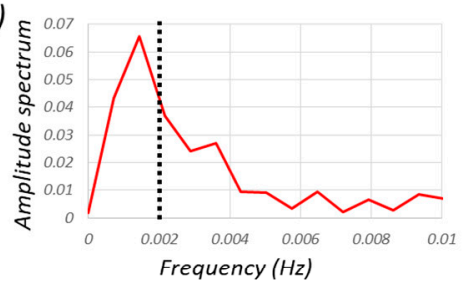

(h)

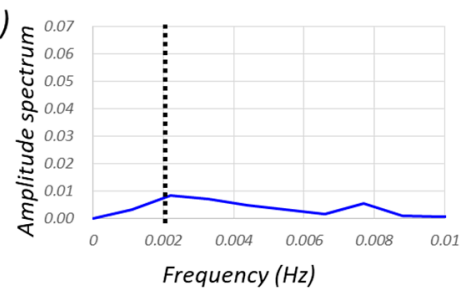

Figure 9. How to achieve frequency analysis, where (a) is the normalized area with respect to time where the approximate curve is shown by the line shown by red color, $(\mathbf{b})$ the curve defined by $S(t)$ $-\mathrm{S}(t)$ approximate curve, and (c) the frequency analysis. The frequency analysis, where (c-e) are under periodic hydrostatic pressure, and $(\mathbf{f}-\mathbf{h})$ are cultured under atmospheric pressure. Three of each of the six original data were carried out.

\section{Discussion}

The on-chip cell incubator proposed in this paper includes two important key words, one of which is "same tme history" and the other is "simultaneous observation". "Same time history" means cells whose initial conditions are exactly the same, including the time for all cells in two chambers. It is a great advantage to completely avoid the influence for the results coming from the time difference among cells, and therefore, we can keep the culture condition the same in both chambers except either under periodic hydrostatic pressure or atmospheric pressure. "Simultaneous observation" allows us to visualize cell behaviors coming from only one parameter, in this work the effect of periodic hydrostatic pressure.

Using the on-chip cell incubator, we could observe how the cells grow by focusing in the first hour for both cultures with and without periodic hydrostatic pressure. The most interesting result under periodic hydrostatic pressure is that cells in growth period increase the projected cell area according to the pressure frequency imparted on the culture liquid. This effect is more enhanced for the cell whose size is bigger. To the best of the authors' knowledge, there was no such size fluctuation of cell size reported in literature under a periodic pressure stimulus. A natural question that comes up is why there has been no report on the frequency-dependent cell size fluctuation so far. Our work is based on an extremely low frequency, $0.002 \mathrm{~Hz}$, while the frequencies of periodic pressure in former works are mostly around $1 \mathrm{~Hz}$, just like heartbeat and breathing [16,18-21]. We believe that several minutes are needed for cells to change size during periodic hydrostatic pressure and it is hard to change the size with a noticeable range under a pressure frequency with around $1 \mathrm{~Hz}$. 


\section{Conclusions}

In the same way that human muscle grows after exercise, it is known that an elastic cell sheet can be obtained by cell culture with periodic hydrostatic pressure. This paper presented simultaneous cell observation by an on-chip cell incubator with and without such a periodic hydrostatic pressure. The periodic pressure with an extremely low frequency of $0.002 \mathrm{~Hz}$ was imparted for one chamber and atmospheric pressure was given for the other one. The experiments were only focused on the first one hour after cell seeding, and significant difference of cell growth were observed. We also found an interesting phenomenon during periodic hydrostatic pressure where the projected areas of the cells increased at the same frequency as the pressure frequency imparted on them. For future work, we plan to test with difference frequencies and see in which frequency the frequency-dependent cell growth disappears.

Supplementary Materials: The following are available online at http:/ / www.mdpi.com/2072-666X/10/2/133/ s1, Video S1: the cell behavior from the point (a) to the point (d) during cell culture, and Video S2: the cell behavior against the pressure value after the point (c).

Author Contributions: All authors conceived and designed the experiments; M.H. performed the experiments; M.H. and C.T., contributed to the data analysis and interpretations; M.H., C.T., and M.K. wrote the paper.

Acknowledgments: This work was partially supported by JSPS KAKENHI Grant-in-Aid for Scientific Research(S) 15H05761, Challenging Exploratory Research 17K18854, Grant-in-Aid for Young Scientists (B) 17K14625, AMED (Japan Agency for Medical Research and Development) 18ek0109240h0002, Nanotech Career-up Alliance (Nanotech CUPAL), and the Ministry of Education via the "Nanotechnology Platform Undertaking". The microfluidic devices were fabricated at Nanotechnology Open Facilities in Osaka University [F-18-OS-0008 S-18-OS-0008].

Conflicts of Interest: The authors declare no conflict of interest.

\section{Appendix A}

In this research, PDMS (X-32-3094-2, Shin-Etsu Chemical Co., Ltd., and Tokyo, Japan) microfluidic chips for cell culture were fabricated by soft lithography technique [26]. The microfluidic device with different channel heights was designed in order to trap cells at the bottom of the chambers, and therefore, two-layer microstructures were fabricated with SU-8 photoresist (SU-8 3050, Microchem Corp., Massachusetts, USA), as shown in Figure A1. The width and length of the flow channels were $300 \mu \mathrm{m}$ and $6000 \mu \mathrm{m}$, respectively, while the diameters of the inlet/outlet and the height of these structures were $1500 \mu \mathrm{m}$ and $50 \mu \mathrm{m}$, respectively. After spin coating for the first layer of photoresist as shown in Figure A1, a baking step for $15 \mathrm{~min}$ at $95^{\circ} \mathrm{C}$ was performed. Figure A1b shows that the SU-8 photoresist was exposed to $200 \mathrm{~mJ} / \mathrm{cm}^{2}$ of UV light with a mask aligner (MA-10, MIKASA CO., LTD, Tokyo, Japan), and then the first layer of photoresist was developed by 5 min baking at $95{ }^{\circ} \mathrm{C}$, as shown in Figure A1c. Figure A1d shows the coating of the second layer of SU-8 photoresist for trapping the cells. The thickness of the second layer was designed as $250 \mu \mathrm{m}$ and is baked for $300 \mathrm{~min}$ at $95{ }^{\circ} \mathrm{C}$ after the coating. The SU-8 photoresist was exposed to $500 \mathrm{~mJ} / \mathrm{cm}^{2}$ of UV light using the mask aligner, as shown in Figure A1e, and baked for another $20 \mathrm{~min}$ at $95^{\circ} \mathrm{C}$ before the second-layer structure was developed, as shown in Figure A1f. The mold was fabricated through the process shown in Figure A1a-f. Afterwards, PDMS mixture with a weight ratio of 10:1 between the base and curing agent was poured onto the mold in a plastic dish. The PDMS was degassed for $30 \mathrm{~min}$ in vacuum desiccators, before being baked for $30 \mathrm{~min}$ at $90{ }^{\circ} \mathrm{C}$. After the PDMS chip was completely cured, the chip was picked up by peeling off the PDMS replicas from the mold as shown in Figure A1g,h. The hardness of the PDMS chip was 80 and was important for suppressing the deformation of the chip for the periodic pressure application of cell culture. Next, holes of $1000 \mu \mathrm{m}$ in diameter for inlet wells and outlet wells in the PDMS sheet were punched as shown in Figure A1i. Finally, a PDMS chip and glass plate were bonded by using a plasma bonding device (CUTE-MP, Femto Science Inc., Gyeonggi-Do, Korea) and baked for $10 \mathrm{~min}$ at $80^{\circ} \mathrm{C}$, as shown in Figure A1j. Figure A1k illustrates the fabricated microfluidic device. 


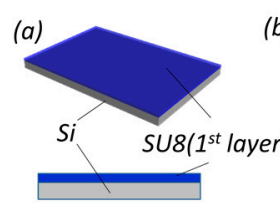

(g)

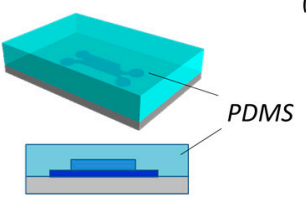

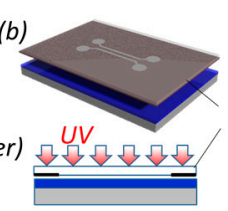

(h)

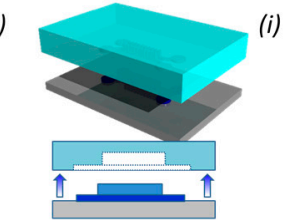

(d)
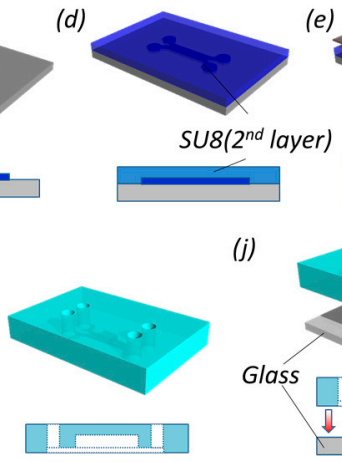

(j)

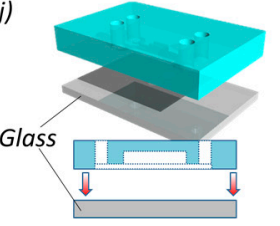

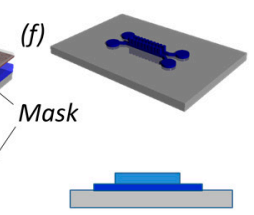

(k)

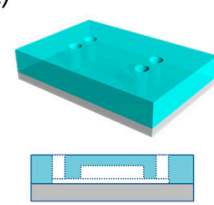

Figure A1. Fabrication of the microfluidic chip. The device consists of inlets, outlets, cell trap chambers. (a-f) Step-by-step photolithography for mold fabrication; (g-k) the step-by-step procedure for PDMS chip fabrication from the mold. PDMS and glass are integrated by plasma bonding.

\section{References}

1. Sies, H.; Berndt, C.; Jones, D.P. Oxidative stress. Annu. Rev. Biochem. 2017, 86, 715-748. [CrossRef] [PubMed]

2. Olagnier, D.; Peri, S.; Steel, C.; Van Montfoort, N.; Chiang, C.; Beljanski, V.; Slifker, M.; He, Z.; Nichols, C.N.; Lin, R.; et al. Cellular Oxidative Stress Response Controls the Antiviral and Apoptotic Programs in Dengue Virus-Infected Dendritic Cells. PLoS Pathog 2014, 10, e1004566. [CrossRef] [PubMed]

3. Veschgini, M.; Gebert, F.; Khangai, N.; Ito, H.; Suzuki, R.; Holstein, T.W.; Mae, Y.; Arai, T.; Tanaka, M. Tracking mechanical and morphological dynamics of regenerating Hydra tissue fragments using a two fingered micro-robotic hand. Appl. Phys. Lett. 2016, 108, 103702. [CrossRef]

4. Murakami, R.; Tsai, C.-H.D.; Ito, H.; Tanaka, M.; Sakuma, S.; Arai, F.; Kaneko, M. Catch, load and launch toward on-chip active cell evaluation. In Proceedings of the 2016 IEEE International Conference on Robotics and Automation (ICRA), Stockholm, Sweden, 16-21 May 2016; pp. 1713-1718.

5. Ohara, K.; Kawakami, D.; Takubo, T.; Mae, Y.; Tanikawa, T.; Honda, A.; Arai, T. Dextrous cell diagnosis using two-fingered microhand with micro force sensor. J. Micro Nano Mechatron. 2012, 7, 13-20. [CrossRef]

6. Horade, M.; Tsai, C.-H.D.; Ito, H.; Kaneko, M. Red Blood Cell Responses during a Long-Standing Load in a Microfluidic Constriction. Micromachines 2017, 8, 100. [CrossRef]

7. Ito, H.; Murakami, R.; Sakuma, S.; Tsai, C.-H.D.; Gutsmann, T.; Brandenburg, K.; Pöschl, J.M.B.; Arai, F.; Kaneko, M.; Tanaka, M. Mechanical diagnosis of human erythrocytes by ultra-high speed manipulation unraveled critical time window for global cytoskeletal remodeling. Sci. Rep. 2017, 7, 43134. [CrossRef] [PubMed]

8. Di Cara, F.; Maile, T.M.; Parsons, B.D.; Magico, A.; Basu, S.; Tapon, N.; King-Jones, K. The Hippo pathway promotes cell survival in response to chemical stress. Cell Death Differ. 2015, 22, 1526-1539. [CrossRef] [PubMed]

9. Seo, K.; Rainer, P.P.; Lee, D.I.; Hao, S.; Bedja, D.; Birnbaumer, L.; Cingolani, O.H.; Kass, D.A. Hyperactive Adverse Mechanical Stress Responses in Dystrophic Heart Are Coupled to Transient Receptor Potential Canonical 6 and Blocked by cGMP-Protein Kinase G Modulation. Circ. Res. 2014, 114, 823-832. [CrossRef] [PubMed]

10. Sakuma, S.; Kuroda, K.; Tsai, C.-H.D.; Fukui, W.; Arai, F.; Kaneko, M. Red blood cell fatigue evaluation based on the close-encountering point between extensibility and recoverability. Lab Chip 2014, 14, 1135. [CrossRef]

11. Teramura, T.; Takehara, T.; Onodera, Y.; Nakagawa, K.; Hamanishi, C.; Fukuda, K. Mechanical stimulation of cyclic tensile strain induces reduction of pluripotent related gene expressions via activation of Rho/ROCK and subsequent decreasing of AKT phosphorylation in human induced pluripotent stem cells. Biochem. Biophys. Res. Commun. 2012, 417, 836-841. [CrossRef]

12. Subramony, S.D.; Dargis, B.R.; Castillo, M.; Azeloglu, E.U.; Tracey, M.S.; Su, A.; Lu, H.H. The guidance of stem cell differentiation by substrate alignment and mechanical stimulation. Biomaterials 2013, 34, 1942-1953. [CrossRef] [PubMed] 
13. Itoh, M.; Nakayama, K.; Noguchi, R.; Kamohara, K.; Furukawa, K.; Uchihashi, K.; Toda, S.; Oyama, J.-I.; Node, K.; Morita, S. Scaffold-Free Tubular Tissues Created by a Bio-3D Printer Undergo Remodeling and Endothelialization when Implanted in Rat Aortae. PLoS ONE 2015, 10, e0136681.

14. Lee, V.K.; Kim, D.Y.; Ngo, H.; Lee, Y.; Seo, L.; Yoo, S.-S.; Vincent, P.A.; Dai, G. Creating perfused functional vascular channels using 3D bio-printing technology. Biomaterials 2014, 35, 8092-8102. [CrossRef]

15. Murphy, S.V.; Atala, A. 3D bioprinting of tissues and organs. Nat. Biotechnol. 2014, 32, 773-785. [CrossRef] [PubMed]

16. Yokoyama, U.; Tonooka, Y.; Koretake, R.; Akimoto, T.; Gonda, Y.; Saito, J.; Umemura, M.; Fujita, T.; Sakuma, S.; Arai, F.; et al. Arterial graft with elastic layer structure grown from cells. Sci. Rep. 2017, 7, 427. [CrossRef] [PubMed]

17. Horade, M.; Kaneko, M.; Tsai, C.D.; Ito, H.; Higashino, N.; Akai, T.; Yokoyma, U.; Ishikawa, Y.; Sakuma, S.; Arai, F. On-Chip Cell Gym. In Proceedings of the 30th IEEE Conference on Micro Electro Mechanical Systems (MEMS2017), Las Vegas, NV, USA, 22-26 January 2017; pp. 603-604.

18. Buschmann, M.D.; Gluzband, Y.A.; Grodzinsky, A.J.; Hunziker, E.B. Mechanical compression modulates matrix biosynthesis in chondrocytelagarose culture. J. Cell Sci. 1995, 108, 1497-1508. [PubMed]

19. Carver, S.E.; Heath, C.A. Increasing extracellular matrix production in regenerating cartilage with intermittent physiological pressure. Biotechnol. Bioeng. 1999, 62, 166-174. [CrossRef]

20. Hall, A.C.; Urban, J.P.G.; Gehl, K.A. The effects of hydrostatic pressure on matrix synthesis in articular cartilage. J. Orthop. Res. 1991, 9, 1-10. [CrossRef]

21. Suh, J.K.; Baek, G.H.; Årøen, A.; Malin, C.M.; Niyibizi, C.; Evans, C.H.; Westerhausen-Larson, A. Intermittent sub-ambient interstitial hydrostatic pressure as a potential mechanical stimulator for chondrocyte metabolism. Osteoarthr. Cartil. 1999, 7, 71-80. [CrossRef]

22. Horade, M.; Mizuta, Y.; Kaji, N.; Higashiyama, T.; Arata, H. Plant-on-a-chip microfluidic-system for quantitative analysis of pollen tube guidance by signaling molecule: Towards cell-to-cell communication study. In Proceedings of the 16th International Conference on Miniaturized Systems for Chemistry and Life Sciences (MicroTAS 2012), Okinawa, Japan, 28 October-1 November 2012; pp. 1027-1029.

23. Yasaki, H.; Yasui, T.; Yanagida, T.; Kaji, N.; Kanaki, M.; Fukuyama, M.; Nagashima, K.; Kawai, T.; Baba, Y. Microfluidic long-pore-channel to highlight bacteria contents. In Proceedings of the 20th International Conference on Miniaturized Systems for Chemistry and Life Sciences (MicroTAS 2016), Dublin, Ireland, 9-13 October 2016; pp. 101-102.

24. Shimizu, K.; Shunori, A.; Morimoto, K.; Hashida, M.; Konishi, S. Development of a biochip with serially connected pneumatic balloons for cell-stretching culture. Sens. Actuators B Chem. 2011, 156, 486-493. [CrossRef]

25. Eyer, K.; Kuhn, P.; Hanke, C.; Dittrich, P.S. A microchamber array for single cell isolation and analysis of intracellular biomolecules. Lab Chip 2012, 12, 765-772. [CrossRef] [PubMed]

26. Anderson, J.R.; Chiu, D.T.; Jackman, R.J.; Cherniavskaya, O.; McDonald, J.C.; Wu, H.; Whitesides, S.H.; Whitesides, G.M. Fabrication of Topologically Complex Three-Dimensional Microfluidic Systems in PDMS by Rapid Prototyping. Anal. Chem. 2000, 72, 3158-3164. [CrossRef] [PubMed]

(C) 2019 by the authors. Licensee MDPI, Basel, Switzerland. This article is an open access article distributed under the terms and conditions of the Creative Commons Attribution (CC BY) license (http:// creativecommons.org/licenses/by/4.0/). 